\title{
La construcción del conocimiento desde la vivencia/experiencia del alumnado de la materia Educación. Desenvolvimiento motor en la formación del docente de Educación Infantil
}

\author{
Building knowledge from the students' experience in the subject Education. \\ Motor development in the Primary Teacher's training \\ A construção do conhecimento a partir da vivência/experiência dos alunos da disciplina \\ Educação. Desenvolvimento motor na formação do docente da Educação Infantil
}

\author{
Jose Pazos Couto, ${ }^{a}$ Maria Venegas Rojas, ${ }^{b}$ \\ Cristian Abelairas Gomez, ${ }^{c}$ Miguel Cons Ferreiro ${ }^{d}$
}

${ }^{a}$ Universidad de Vigo, Vigo, España.

Teléfono: (34) 627589680. Correo electrónico: chema3@ gmail.com

bUniversidad de A Coruña, La Coruña, España.

Teléfono: (34) 693778472. Correo electrónico: ma.jose0602@gmail.com

'Universidad Europea de Atlántico, Santander, España.

Teléfono: (34) 618824297. Correo electrónico: cristianabelairasgomez@gmail.com

${ }^{\mathrm{d}}$ Universidad de Vigo, Vigo, España.

Teléfono: (34) 639106231. Correo electrónico: miguelcons@gmail.com

\section{RESUMEN}

El presente artículo muestra la experiencia obtenida en las clases de la asignatura Educación. Desarrollo motor en el grado de Educación infantil, en la visión y percepción de los alumnos de esta materia. Además de describir el modelo pedagógico y el enfoque que el profesor utiliza para el desarrollo de la misma. Se utiliza un cuestionario de valoración final de la materia en el que se quiere conocer el cómo vivencian este proceso de aprendizaje, con cuestiones como la eficacia de las clases teóricas y prácticas, los aspectos positivos y negativos de la misma, los aprendizajes obtenidos, entre otras. Como resultado aparece la buena acogida entre el alumnado de la utilización de esta metodología para la realización y obtención de su propio aprendizaje.
\end{abstract}

Palabras clave: formación de profesorado, didáctica, aprendizaje, motricidad.

\section{ABSTRACT}

This article shows the experience gained in the class Education. Motor development in the Primary Teacher's training, from the students' point of view about this subject. Besides it describes the teaching model and the approach the teacher uses to develop this class. An evaluation survey about the subject is used to understand how this learning process is experienced, with questions about the efficacy of both the theoretical and practical classes, the positive and negative aspects of the subject, the learnings gained, etc. Having as a result a good reception from the student body in the usage of this methodology to develop and obtain their own learnings.

Key words: faculty's training, didactics, learning, human movement. 


\section{RESUMO}

Apresenta-se a experiência obtida em aulas da disciplina Educação. Desenvolvimento Motor na graduação em Educação Infantil, a partir da visão e percepção dos alunos da disciplina. Além disso, descreve-se o modelo pedagógico e o enfoque adotado pelo professor para o desenvolvimento das atividades. Utiliza-se um questionário de avaliação final da disciplina em que se quer saber como são vivenciados os processos de aprendizagem, com questões sobre a eficácia das aulas teóricas e práticas, os aspectos positivos e negativos, as aprendizagens obtidas, entre outras. Tem-se como resultado a boa receptividade de esta metodologia adotada pelos alunos para a realização e obtenção de sua própria aprendizagem.

Palavras chave: formação do professorado, didática, aprendizagem, motricidade.

\section{INTRODUCCIÓN}

La formación inicial de docentes es un tema recurrente cuando se piensa en la calidad de la educación en los diferentes niveles y etapas del sistema educativo. En los últimos años, en España, con la implantación de los grados universitarios esta formación ha adquirido un estatus similar al de los demás grados universitarios, y también como consecuencia del Plan Bolonia se están estimulando diferentes formas de transmisión y creación de conocimiento, partiendo de la premisa de que cada grupo de profesores que conforma el equipo docente de una titulación debe plantearse cuál/es de las competencias de dicha titulación puede desarrollar desde sus respectivas áreas y cómo va a hacerlo (Ion y Cano, 2012). Es vital en este planteamiento conocer las percepciones de los agentes implicados, pues el resultado depende en gran medida de su aceptación por parte de estos, sobre todo cuando tratamos de innovar en el ámbito de la praxis docente.

El planteamiento parte de comprender que la teoría solo se vincula decisivamente a un proceso de transformación a través de la unidad dialéctica teoría-práctica (Sérgio, 2008), y es a partir de ahí que se generan los aprendizajes significativos, en la dinámica del aprender con la praxis de la acción relacionada con el marco conceptual. Esta idea se refuerza más cuando hablamos de desarrollo humano, o de procesos de interacción entre personas en los que se pretende que se produzcan aprendizajes.

Damasio (2000) nos dice que la razón entraña siempre sentimiento, partiendo de esta idea justificamos que el dúo comprensión-aprendizaje van ligados a la razón, y todo ello se mezcla en el día a día del aula, que convierte el proceso formativo en una realidad compleja que nos permite ir más allá del entorno académico y poder filtrar así los procesos de aprendizaje a la vida de las personas, tratando de hacer realidad las dinámicas de concientización de las que nos habla Paulo Freire (2002).

El proceso de formación en la educación superior debe hacernos conscientes de que en esta sociedad del teatro de las apariencias, el parecer prima sobre el ser. Todos lo sabemos, pero todos cedemos (Morin y Hulot, 2008). Y es desde la educación desde donde se puede cambiar esta situación.

Igualmente, al hablar de la educación superior en la formación de profesorado, buscamos formar profesionales que partan de su práctica para confirmar o modificar sus teorías, sin olvidar estas, promoviendo una actitud de búsqueda para provocar aprendizajes a partir de esta experiencia (Sales, 2006). Hablamos de una educación por competencias con un enfoque humanista, que partiendo de las experiencias del pasado permita adaptarse a las circunstancias del mundo actual (Alejo et al., 2013). 
Otra de las líneas básicas de trabajo es la de una metodología que se apoye en diversos momentos (teórico-prácticos) en el aprendizaje cooperativo, que necesariamente es una fuente de aprendizajes autónomos en el alumnado y, por qué no, en el profesorado. Así, la diversidad de contenidos trabajados mediante aprendizaje cooperativo afianza la idea de que cualquier contenido puede ser enseñado mediante esta metodología (VelázquezCallado, 2015).

La línea de pensamiento/trabajo pasa por la reflexión sobre el proceso docente, de cara a construir conocimiento en el alumnado, partiendo de las experiencias y vivencias de todos los implicados en el proceso educativo, de cara a conseguir aprendizajes autónomos a partir de estas.

En todo este proceso la evaluación tiene un valor primordial, no como calificación ni como trampa para profesor o alumno, sino como proceso paralelo al aprendizaje, como nos dicen Herraiz, Miño y Piqué (2014) hemos de pretender que la evaluación sea un proceso compartido en el que todos puedan reflexionar y cuestionarse si han cumplido sus expectativas.

\section{EL PLANTEAMIENTO}

Partimos de un planteamiento abierto, en el que el alumno es el protagonista de sus aprendizajes, y no como tradicionalmente ha sido entendido dentro del contexto académico, como una adquisición de la información por parte del alumno, de determinados temas ubicados en un currículum predeterminado (Toro, 2006), a través de procesos memorísticos. Nosotros consideramos básica la vivencia de la praxis y la experiencia necesaria de la acción, ahora bien ¿cómo lo vamos a articular?

Un componente esencial de este método de aprendizaje es el debriefing (Maestre y Rudolph, 2015), que se basa en la conversación grupal para analizar una situación real o simulada, en la que los participantes analizan sus acciones y reflexionan sobre el papel de los procesos de pensamiento, las habilidades psicomotrices y los estados emocionales para mejorar o mantener su rendimiento en el futuro, o el aprendizaje dialógico del que nos hablan Castro et al. cuando destacan "la importancia de la interacción para que se produzcan procesos de transformación individual y social a través del diálogo, ya que la construcción del conocimiento se inicia en un plano intersubjetivo/social que va concretándose en una construcción personal" (2014: 174).

Siguiendo estas premisas nos hemos planteado utilizar estos conceptos como herramienta principal en el desarrollo de las sesiones de la materia.

Antes de continuar, veamos cómo se encuentra estructurada la materia:

1. Es una materia de $1^{\circ}$ curso de grado en Educación Infantil, que se imparte en el segundo semestre y que consta de 6 créditos ECTS.

2. La estructura de la materia consta de dos partes bien diferenciadas en el aula, que son la parte práctica (30 horas) en la que el grupo de divide en Grupos de Tipo B (entre 15-20 alumnos) y la parte teórica de la materia en la que hay un único grupo que consta de unos 50 alumnos.

3. La evaluación, a rasgos generales, sería como sigue:

a. Habrá la modalidad de evaluación continua, en la que la asistencia es obligatoria.

b. También habrá la modalidad estándar, en la que el trabajo y proyecto se corresponderá 
Estudios Pedagógicos XLI, N Especial: 181-192, 2015

LA CONSTRUCCIÓN DEL CONOCIMIENTO DESDE LA VIVENCIA/EXPERIENCIA DEL ALUMNADO DE LA MATERIA EDUCACIÓN. DESENVOLVIMIENTO MOTOR EN LA FORMACIÓN DEL DOCENTE DE EDUCACIÓN INFANTIL

con una exposición práctica, demostración de competencia motriz y presentación de trabajos de campo.

c. En la segunda convocatoria se guardarán las calificaciones obtenidas que habían tenido por lo menos una nota de cinco (5).

d. El alumnado deberá superar cada parte de la asignatura de la siguiente manera:

i. Parte teórica: Examen tipo test.

ii. Parte práctica:

a) si siguió evaluación continua durante el curso deberá presentar un trabajo asignado por el profesor.

b) si no siguió evaluación continua deberá hacer un examen de preguntas abiertas.

Las metodologías utilizadas fueron:

Tabla 1. Metodologías utilizadas durante el desarrollo de la materia

\begin{tabular}{|l|l|}
\hline Sesión magistral & Sesiones magistrales en el aula o en los espacios que requieran las materias \\
\hline Trabajos de aula & $\begin{array}{l}\text { El alumnado preparará un trabajo orientado por el profesorado en base a los } \\
\text { contenidos de la materia. }\end{array}$ \\
\hline Debates & $\begin{array}{l}\text { Debates de aula en base a temas de la materia o de actualidad que tengan que } \\
\text { ver con el desarrollo motor. }\end{array}$ \\
\hline $\begin{array}{l}\text { Presentaciones } \\
\text { exposiciones }\end{array}$ & $\begin{array}{l}\text { En grupos y orientados por el profesorado, se prepara y expone un trabajo } \\
\text { vinculado a la motricidad infantil. Se trata de una propuesta práctica de } \\
\text { intervención que debe estar fundamentada en base a los contenidos teóricos } \\
\text { yestrategias prácticas. }\end{array}$ \\
\hline $\begin{array}{l}\text { Prácticas de } \\
\text { laboratorio }\end{array}$ & $\begin{array}{l}\text { Prácticas organizadas por el profesorado, vinculadas a los contenidos de la } \\
\text { materia. }\end{array}$ \\
\hline $\begin{array}{l}\text { Eventos docentes } \\
\text { y/o divulgativos }\end{array}$ & $\begin{array}{l}\text { Asistencia a congresos, seminarios o conferencias vinculadas a los } \\
\text { contenidos de la materia. }\end{array}$ \\
\hline
\end{tabular}

La dinámica de la materia, más allá de la estructura formal, fue enfocada a que el alumnado integrara las competencias básicas de la materia que eran:

1. Conocer los fundamentos de la motricidad humana con especial incidente en la etapa de los 0 a 6 años.

2. Entender los diferentes modelos y escuelas del desarrollo psicomotor.

3. Saber estimular las habilidades motrices genéricas a través de tareas y propuestas prácticas.

4. Distinguir e identificar las conductas motrices que se van produciendo en el desarrollo del niño. Identificar las conductas reflejas y el control motor reflejo.

5. Entender los vínculos de la motricidad con las emociones y saber estimularlos mediante tareas lúdico-motrices.

6. Reconocer la diversidad y la interculturalidad a través de tareas motrices en la etapa infantil.

Entendemos la formación humana como aquella posibilidad que solo es privilegio del ser humano, ya que este puede trascender la mera necesidad de supervivencia hacia el deseo 
por vivir y alcanzar la felicidad (Orrego, 2007) y transformar así su realidad cotidiana para la construcción y mejora de su contexto social: esa búsqueda guía nuestro quehacer diario en el aula.

¿Cómo articulamos esta forma de entender el proceso docente y lo convertimos en la realidad del aula? Esta es una búsqueda constante, que vamos afinando año a año. Durante este curso le planteamos al alumnado diferentes propuestas de cara a que pudiesen construir sus aprendizajes.

En la parte práctica de la materia se distribuyeron catorce temas que se dieron a elegir de forma libre a los estudiantes para hacer un breve trabajo de desarrollo a elaborar en parejas para los que se dio un guion de elaboración y que además debería incluir el desarrollo de cinco sesiones de una hora cada una, que en la medida de lo posible llevaríamos a cabo en el aula.

Antes de realizar las exposiciones en el aula, se lleva a cabo en horario de tutorías un análisis-revisión previo de lo que los alumnos pretender realizar en el aula, para garantizar que la exposición será sobre el tema en cuestión, para obtener una opinión por parte del profesor y que este pueda realizar aportaciones, así como para concretar aspectos como el lugar donde se realizará la sesión, materiales necesarios y otras dudas que pudiesen tener los alumnos, esto se realiza como mínimo la semana anterior a la exposición del tema en clase.

La dinámica de las prácticas consistía en buscar el protagonismo del alumnado, cada día de prácticas se realizaba la exposición de dos temas y se realizaban en el aula dos exposiciones prácticas que desarrollaban los alumnos. En estas prácticas el profesor participaba como un alumno más, sin cortar en ningún momento la dinámica de la misma bajo ningún concepto (salvo accidente o injerencia externa), solo asumía el rol de un alumno más.

Una vez hechas las dos exposiciones diarias, el grupo se sienta a conversar sobre lo sucedido en el aula, siguiendo una estructura que sí marca el profesor y que es la que sigue:

1. Torbellino de ideas sobre lo que hemos hecho en clase, de forma muy dinámica, sin importar que se repitan conceptos o ideas y sin interrupción.

2. ¿Cómo me he sentido? Cada alumno habla libremente sobre cómo se ha sentido en el aula, sin réplica y sin interrupción.

3. ¿Qué me ha gustado y que no me ha gustado de la case de hoy?

4. ¿Qué cambiaría de la clase de hoy?

5. Los alumnos que han realizado la exposición hablan sobre cómo se han sentido en el aula, lo que ellos cambiarían a posteriori, en qué han acertado y sobre las decisiones que han tenido que ir tomando a lo largo de su exposición en función de cómo iban percibiendo al grupo.

A partir de este momento, se establece un debate libre moderado por el profesor, en el que analizamos qué ha pasado en el aula, tanto a nivel de por qué nos hemos sentido así, y sobre cómo mejoraríamos la dinámica del aula con el objetivo de que fuese más lúdica sin perder de vista el sentido de la misma. En este mismo debate, pero al final, sí interviene el profesor de forma activa, y hace un análisis también de sus vivencias, así como de lo que ha sucedido en el aula.

Es de destacar que se pone mucho énfasis en que el alumnado no se sienta evaluado por el profesor, en que no existe una calificación numérica por medio, las críticas, reflexiones y aportaciones realizadas tanto por el alumnado como por el profesor buscan una mejora de la formación docente y una toma de consciencia sobre la participación en el aula. 
El hecho de que el profesor participe como un alumno más busca, entre otros objetivos, mostrar que el profesor es y debe ser un elemento más de la formación docente y no es un simple observador, la participación ayuda a la percepción de cercanía del mismo, en palabras de Jarauta y Medina (2012) la calidad del profesor en las aulas no se reduce únicamente al dominio de los contenidos de su área de especialización. Necesita de otros saberes: pedagógicos, curriculares, contextuales, proposicionales y prácticos y de la habilidad de manipularlos, transformarlos y conectarlos para dar una respuesta adecuada a cada situación de aula.

En la parte teórica de la materia, en la que está todo el grupo en clase (los cuatro grupos B que componen el grupo A) el enfoque es diferente, por afrontar una situación y contextos distintos. En el grupo A la base del trabajo diario es la elaboración de mapas mentales por parte del alumnado antes de la clase, con lo que se persigue que vengan con el tema trabajado al aula a través del material que el profesor les facilita a través del FAITIC (plataforma virtual de seguimiento de la asignatura que facilita la Universidad de Vigo como apoyo a la docencia).

A partir del material facilitado y de los mapas elaborados por el alumnado se establece el diálogo en el aula, partiendo de la aclaración de las dudas surgidas en la lectura/trabajo del material facilitado, y se continúa una conversación entre el profesor y los alumnos que trata de enriquecer el conocimiento adquirido, tratando de ejemplificar al máximo los temas vistos, buscando situaciones vividas por el alumnado o poniendo en situación al alumnado de hipotéticas situaciones que puedan surgir en la realidad.

La intención es provocar el conocimiento vivenciado a partir de la puesta en común de situaciones y experiencias. Al igual que en la parte práctica, buscamos provocar que el alumno sea parte activa de sus aprendizajes, a través del trabajo y de la verbalización de la experiencia, teniendo en cuenta la opinión/pensamiento del alumnado, elaborado a partir de su trabajo y vivencias. En palabras de Ayala et al. (2011):

uno de los primeros retos del profesor está en aprender a tolerar el silencio en vez de intervenir para llenar los vacíos durante las discusiones de los estudiantes, y más aún reconocer que es necesario mantener el silencio durante periodos prolongados para que sean los propios estudiantes quienes descubran asuntos importantes y reformulen sus ideas (2011: 349).

Teniendo en cuenta que cuando hablamos de práctica no hablamos solo de intervención educativa en el aula, ni únicamente de esa intervención real; detrás del diseño de programas y proyectos, tanto escolares como de intervención ante diferentes grupos sociales, debe existir esa intencionalidad y debe quedar plasmada esa búsqueda del desarrollo personal desde el punto de vista de la motricidad y que den así el paso de la "instrucción al desarrollo" (Pazos, 2006).

\section{LOS INSTRUMENTOS DE ANÁLISIS}

Los instrumentos que hemos utilizado para analizar la realidad del aula y del desarrollo de la asignatura han sido los mapas mentales, los registros de participación en el aula, el diario del profesor y el cuestionario de evaluación Final, para lo que hemos adaptado el Cuestionario de Valoración (CV) utilizado por Trigo (2001). 
En nuestro cuestionario de valoración final se recoge información a través de 7 preguntas de cómo fue el aprendizaje a lo largo del curso, tanto en la parte práctica de la materia como en la teórica (cómo percibió la materia), como en el cognoscitivo (qué aprendió de los contenidos de la asignatura y de la aplicación didáctica que se llevó a cabo) o en el de futuro profesional (para qué te va a servir), además de una valoración de la participación del profesor y una valoración propia del alumno sobre su participación, así como una valoración del trabajo realizado.

Tabla 2. Contenido del cuestionario de evaluación

\begin{tabular}{|c|c|c|c|}
\hline \multicolumn{4}{|c|}{ CONTENIDO DEL C.V. } \\
\hline $\mathrm{N}^{\mathrm{o}}$ Ítem & $\mathrm{N}^{\circ}$ Variables & Finalidad & Tipo \\
\hline 1 & 6 & $\begin{array}{l}\text { Conocer de manera global la valoración de } \\
\text { las clases teóricas del curso en } 6 \text { aspectos }\end{array}$ & Escala 1-6 respuesta única. \\
\hline 2 & 6 & $\begin{array}{l}\text { Conocer de manera global la valoración de } \\
\text { las clases prácticas del curso en } 6 \text { aspectos. }\end{array}$ & Escala 1-6 respuesta única. \\
\hline 3 & 1 & Percepción sobre lo aprendido en el curso. & Respuesta única (sí/no). \\
\hline 4 & 1 & $\begin{array}{l}\text { Utilidad de la experiencia de cara a } \\
\text { reproducirla en el futuro. }\end{array}$ & Respuesta única (sí/no) \\
\hline 5 & 1 & $\begin{array}{l}\text { Aplicación futura de aplicación como } \\
\text { docente. }\end{array}$ & Respuesta única (sí/no) \\
\hline 6 & 1 & Valoración de la actuación del Profesor. & Escala 1-10 respuesta única \\
\hline 7 & 1 & Valoración de la actuación del alumno. & Escala 1-10 respuesta única \\
\hline
\end{tabular}

En los ítems 3, 4 y 5 se solicita al alumnado la justificación de la respuesta, para conocer el sentido de la misma.

Este último instrumento es el que hemos utilizado para extraer los datos concluyentes sobre el resultado de esta experiencia docente.

\section{EL GRUPO DE TRABAJO Y LAS CONDICIONES}

El trabajo ha sido realizado con alumnos de la Materia "Educación. Desenvolvimiento Motor", del segundo semestre del primer curso del Grado en Educación Infantil de la Universidad de Vigo (España), en la que estaban matriculados un total de 49 alumnos, de los cuales en la muestra de datos final han participado un total de 38 alumnos, (36 alumnas y 2 alumnos), todos ellos de primer curso del grado en Educación Infantil y matriculados por primera vez en la materia.

La docencia se desarrolló de forma habitual en las instalaciones de la Facultad de Ciencias de la Educación y del Deporte de Pontevedra, llevándose a cabo las clases prácticas de forma habitual en el pabellón y las teóricas en un aula. 
Estudios Pedagógicos XLI, Nº Especial: 181-192, 2015

LA CONSTRUCCIÓN DEL CONOCIMIENTO DESDE LA VIVENCIA/EXPERIENCIA DEL ALUMNADO DE LA MATERIA EDUCACIÓN. DESENVOLVIMIENTO MOTOR EN LA FORMACIÓN DEL DOCENTE DE EDUCACIÓN INFANTIL

\section{RESULTADOS Y DISCUSIÓN}

En un primer análisis descriptivo, podemos comprobar que la pregunta 1 sobre el estilo de las clases teóricas presenta una media en todas las preguntas superior a la media de la escala empleada, aunque es llamativo que el valor del diseño de las sesiones teóricas, a pesar de su diseño, sean los que han obtenido peores valores, con solo un 4,243; en cuanto a la desviación, merece ser destacado que la eficacia del método utilizado es la pregunta con la mayor desviación $(1,1211)$ como podemos ver en a Tabla 3.

Tabla 3. Media, Desviación Típica y error de la Desviación Típica de la pregunta uno

\begin{tabular}{|l|c|c|c|}
\hline \multicolumn{1}{|c|}{ Ítems preguntados $(\mathrm{n}=37)$} & Media & D. Típica & Error DT \\
\hline De muy ineficaz a muy eficaz & 4,514 & 1,1211 &, 1843 \\
\hline De muy aburrido a muy divertido & 4,568 &, 8673 &, 1426 \\
\hline De muy autoritario a muy democrático & 4,514 &, 9013 &, 1482 \\
\hline De muy pasivo a muy activo & 4,892 &, 9364 &, 1539 \\
\hline De muy rutinario a muy creativo & 4,243 &, 9833 &, 1617 \\
\hline De muy tradicional a muy novedoso & 4,811 &, 9672 &, 1590 \\
\hline
\end{tabular}

En la pregunta 2 del cuestionario, que versaba sobre las sesiones prácticas y cuyos datos obtenidos se presentan en la tabla 4, podemos observar que los valores medios tiene una mejor calificación que los teóricos (ver Tabla 3), y a su vez presentan tanto una desviación típica como un error en la misma, más pequeño. Merece ser destacada la media del ítem de diversión en las clases $(5,892)$, que es la más elevada junto con la actividad en las sesiones $(5,865)$, que a su vez presentan la menor desviación típica $(0,3933$ y 0,3466$)$ y los menores errores $(0,0647$ y 0,0570$)$.

Tabla 4. Media, Desviación Típica y error de la Desviación Típica de la pregunta 2

\begin{tabular}{|l|c|c|c|}
\hline \multicolumn{1}{|c|}{ Ítems preguntados $(\mathrm{n}=37)$} & Media & D. Típica & Error DT \\
\hline De muy ineficaz a muy eficaz & 5,811 &, 6163 &, 1013 \\
\hline De muy aburrido a muy divertido & 5,892 &, 3933 &, 0647 \\
\hline De muy autoritario a muy democrático & 5,730 &, 4502 &, 0740 \\
\hline De muy pasivo a muy activo & 5,865 &, 3466 &, 0570 \\
\hline De muy rutinario a muy creativo & 5,757 &, 4947 &, 0813 \\
\hline De muy tradicional a muy novedoso & 5,676 &, 6260 &, 1029 \\
\hline
\end{tabular}


Comparando las medias de las mismas preguntas en función del tipo de sesión, podemos comprobar que media obtenida en las sesiones prácticas es superior a las sesiones teóricas, destacando la mayor diferencia en la pregunta de creatividad $(1,514)$, como se puede comprobar en la tabla 5.

Tabla 5. Comparación de las medias obtenidas en la pregunta 1 y la pregunta 2

\begin{tabular}{|l|c|c|}
\hline \multicolumn{1}{|c|}{ Ítems preguntados $(\mathrm{n}=37)$} & Media Pregunta 1 & Media Pregunta 2 \\
\hline De muy ineficaz a muy eficaz & 4,514 & 5,811 \\
\hline De muy aburrido a muy divertido & 4,568 & 5,892 \\
\hline De muy autoritario a muy democrático & 4,514 & 5,730 \\
\hline De muy pasivo a muy activo & 4,892 & 5,865 \\
\hline De muy rutinario a muy creativo & 4,243 & 5,757 \\
\hline De muy tradicional a muy novedoso & 4,811 & 5,676 \\
\hline
\end{tabular}

En la pregunta 3 se le solicita al alumnado que destaque aspectos positivos y negativos sobre la metodología seguida. Los aspectos positivos más destacados son la cercanía del profesor, el aprender jugando y que las clases prácticas sean tan interactivas y el sentirse profesor en la práctica a través de la vivencia como docentes de la misma. En cuanto a lo negativo, destacan que se les exige demasiado trabajo en el global de la asignatura y el que las clases teóricas a veces se hacían muy pesadas.

En relación a las preguntas 4 y 5 , que cuestionan el aprendizaje de algo y si es una experiencia útil en el aprendizaje respectivamente, merece ser destacado que la totalidad de la muestra respondió que sí aprendieron y que fue una experiencia útil. En cuanto a lo que aprendieron, destacan lo siguiente: que han podido vivenciar una nueva forma de entender la docencia universitaria, que conocieron una nueva forma de aprender y que aprendieron a realizar trabajos adaptándose a una normativa establecida. Además, destacan el conocer e interactuar con los compañeros y el profesor de una forma diferente y una nueva forma de enseñar o de entender el rol del maestro de Educación Infantil.

En relación a la utilidad del método, refieren que durante las clases prácticas se aprende de una forma divertida y que la teoría se muestra de una forma diferente. También destacan que pueden ver cómo se aplican conceptos teóricos en la práctica y que es una forma diferente de aprender.

Merece ser destacado que la pregunta 5 presentaba un ítem para saber si aplicarían el mismo método en su futura labor docente, y el $92 \%$ indicó que sí la utilizarían. Y casi en su totalidad consideran que es una forma divertida de aprender y motivante para el alumno.

En la pregunta 6, que califica la labor docente, la media obtenida es de 8,461; mientras que la pregunta 7 -que es una calificación propia de su nivel de participación, una autocalificación- es de 8,0533, con una muestra de 38 registros. Las causas que atribuyen a esa calificación (pregunta 6) son: la cercanía del profesor, el esfuerzo del mismo en que 
Estudios Pedagógicos XLI, N Especial: 181-192, 2015

LA CONSTRUCCIÓN DEL CONOCIMIENTO DESDE LA VIVENCIA/EXPERIENCIA DEL ALUMNADO DE LA MATERIA EDUCACIÓN. DESENVOLVIMIENTO MOTOR EN LA FORMACIÓN DEL DOCENTE DE EDUCACIÓN INFANTIL

aprendan los alumnos y el que se preocupe en la preparación de las clases. Las causas atribuidas a la pregunta 7 se deben a su trabajo realizado en clases, la asistencia regular y su implicación durante las mismas. La opinión que emana sobre la valoración del profesor está en la línea de lo que nos destacan Jarauta y Medina (2012) en cuanto a lo esperado y deseado de la actuación del profesor.

La pregunta 8, que se puntúa según el trabajo realizado en el cuatrimestre, obtiene una media de 8,250 en las 37 respuestas válidas, que atribuyen a los mismos factores que en la pregunta número 7. Dado lo que nos dicen de las preguntas 7 y 8 , podemos destacar que sí hemos conseguido una comprensión de la evaluación en la línea de lo que nos dicen Herrais et al. (2014).

Las correlaciones existentes entre las sesiones teóricas y las prácticas se presenta en la Tabla 6.

Tabla 6. Correlaciones entre las sesiones teóricas y prácticas

\begin{tabular}{|l|c|c|}
\hline & Correlación & Sig. \\
\hline De muy ineficaz a muy eficaz &,- 057 &, 740 \\
\hline De muy aburrido a muy divertido &, 103 &, 542 \\
\hline De muy autoritario a muy democrático &, 078 &, 648 \\
\hline De muy pasivo a muy activo &, 125 &, 461 \\
\hline De muy rutinario a muy creativo &,- 046 &, 786 \\
\hline De muy tradicional a muy novedoso &, 217 &, 197 \\
\hline
\end{tabular}

En cuanto a las prueba $t$, destacamos que existe una valoración más positiva de las sesiones prácticas que de las teóricas, siendo esta valoración estadísticamente significativa.

Tabla 7. Resultados de la prueba $\mathrm{T}$

\begin{tabular}{|l|c|c|c|}
\hline Ítems preguntados (n=37) & $\mathrm{T}$ & $\mathrm{Gl}$ & Sig. (bilateral) \\
\hline De muy ineficaz a muy eficaz & $-6,026$ & 36 &, 000 \\
\hline De muy aburrido a muy divertido & $-8,809$ & 36 &, 000 \\
\hline De muy autoritario a muy democrático & $-7,582$ & 36 &, 000 \\
\hline De muy pasivo a muy activo & $-6,184$ & 36 &, 000 \\
\hline De muy rutinario a muy creativo & $-8,212$ & 36 &, 000 \\
\hline De muy tradicional a muy novedoso & $-5,099$ & 36 &, 000 \\
\hline
\end{tabular}


Podemos destacar que en las tablas 6 y 7 se destaca una diferencia entre lo sucedido en las clases teóricas y las clases prácticas, teniendo mucha más aceptación estas últimas, lo que nos indica que si queremos mejorar la calidad docente, hemos de potenciar o buscar nuevas estrategias para la parte teórica de la materia, pues ya sea debido a la falta de experiencia suficiente utilizando estas metodologías por parte del profesor, o bien a lo "diferente" que han percibido los alumnos la docencia, su percepción, si bien no ha sido mala, no ha estado a la altura de las clases prácticas, lo que indica que se deberían llevar a cabo cambios o modificaciones en este sentido.

En cierta medida, de los resultados obtenidos en la valoración del alumnado, podemos destacar que sí han percibido la unión teoría práctica de la que nos hablan diversos autores (Alejo et al., 2013; Morin y Hulot, 2008; Sales, 2006; Sérgio, 2008; Velázquez, 2015).

\section{CONCLUSIONES}

A modo de conclusión destacaremos la aceptación satisfactoria que tiene la aplicación de una metodología activa por parte del alumnado de cara a su formación como futuros docentes, que va más allá de la vivencia y se proyecta de forma positiva hacia su desarrollo profesional.

Además, existe una percepción de que con esta metodología se consiguen los aprendizajes esperados y una fuerte implicación del alumnado en el desarrollo de la docencia. El desarrollar una metodología en la que el alumno es parte activa de la misma facilita el debate y el protagonismo del alumnado en la elaboración de aprendizajes autónomos y colaborativos.

La presencia de sesiones prácticas muestra al alumnado una "alternativa posible" de cara a su futuro profesional como docentes de Educación Infantil, en la que el niño aprenda jugando, conociendo y vivenciando su propia realidad, en la que la motricidad tiene un papel destacado, facilitando su desarrollo personal.

\section{REFERENCIAS BIBIOGRÁFICAS}

Alejo, A., Reyes, V., \& Rodríguez, V. (2013). La nueva generación de estudiantes y el actuar docente, bajo un proceso de enseñanza-aprendizaje competente. Revista iberoamericana para la investigación y el desarrollo educativo, 10. Recuperado desde http://ride.org.mx/1-11/index.php/ RIDESECUNDARIO/article/view/384/376

Ayala, R., Messing, H. B., \& Toro, S. (2011). El sentido didáctico del "Aprendizaje Basado en Problemas" en la educación médica. Educación Médica Superior, 25(3) 344-351.

Castro, M., Gómez, A., \& Macazaga, A. (2014). Aprendizaje dialógico y grupos interactivos en Educación Física. Retos, 25, 174-179.

Damasio, A. (2000). O misterio da consciencia (1 ${ }^{\mathrm{a}}$ ed., Vol. 1). Sao paulo: Companhia das letras.

Freire, P. (2002). Concientización: Teoría y práctica de una educación liberadora (12ªed., Vol. 1). Buenos Aires: Galerna-Búsqueda de Ayllu.

Herraiz, F., Miño, R., \& Piqué, B. (2014). Una reflexión en torno a la evaluación para el aprendizaje autónomo. Revista del Congrés Internacional de Docència Universitària i Innovació, 2. Recuperado desde http://www.cidui.org/revistacidui/index.php/cidui/article/view/524/505

Ion, G., \& Cano, E. (2012). La formación del profesorado universitario para la implementación 
de la evaluación por competencias. Educación XXI, 15(2), 249-270.

Jarauta, B., \& Medina, J. L. (2012). Saberes docentes y enseñanza universitaria. Estudios sobre educación, 22, 179-198.

Orrego, J.F. (2007). La pedagogía como reflexión del ser en la educación. Revista Latinoamericana de estudios educativos, 3(1), 27-39.

Maestre, J., \& Rudolph, J. (2015). Teorías y estilos de debriefing: el método con buen juicio como herramienta de evaluación formativa en salud. Revista española de Cardiología, 68(4), 282-285.

Morin, E., \& Hulot, N. (2008). El año I de la era ecológica. Barcelona: Paidos.

Pazos, J. (2006). Una visión teórica y metodológica de la motricidad. Pensamiento educativo, 38, 33-45.

Sales, A. (2006). La formación inicial del profesorado ante la diversidad: una propuesta metodológica para el nuevo espacio europeo de educación superior. Revista Interuniversitaria de Formación del Profesorado, 20(3), 201-217.

Sergio, M. (2008). Textos Insólitos ( $1^{\mathrm{a}}$ ed. Vol. 1). Lisboa: Instituto Piaget.

Toro, S. (2006). Conocimiento y motricidad humana, aproximación y desafíos. Pensamiento educativo, 38, 62-74.

Trigo, E. (Coord.). (2001). Motricidad creativa. Una forma de investigar. A Coruña: Universidade da Coruña.

Velázquez, C. (2015). Aprendizaje cooperativo en Educación Física: Estado de la cuestión y propuesta de intervención. Retos, 28, 234-239. 